\title{
Tracking in Nonlinear Differential-Algebraic Control Systems with Applications to Constrained Robot Systems*
}

\author{
HARIHARAN KRISHNAN $\dagger$ and N. HARRIS MCCLAMROCH $\ddagger$ \\ Nonlinear control systems modeled by differential-algebraic equations are \\ studied. A tracking problem associated with the system is solved and its \\ applications to force and position control in robotics are presented.
}

Key Words-Nonlinear control; differential-algebraic equations; force control; constrained robot systems.

\begin{abstract}
We consider the design of a feedback control law for control systems described by a class of nonlinear differential-algebraic equations so that certain desired outputs track given reference inputs. The nonlinear differential-algebraic control system being considered is not in state variable form. Assumptions are introduced and a procedure is developed such that an equivalent state realization of the control system described by nonlinear differential-algebraic equations is expressed in a familiar normal form. A nonlinear feedback control law is then proposed which ensures, under appropriate assumptions, that the tracking error in the closed loop differential-algebraic system approaches zero exponentially. Applications to simultaneous contact force and position tracking in constrained robot systems with rigid joints, constrained robot systems with joint flexibility, and constrained robot systems with significant actuator dynamics are discussed.
\end{abstract}

\section{INTRODUCTION}

WE CONSIDER a class of control systems which is described by nonlinear differential and algebraic equations of the form

$$
\begin{gathered}
\dot{x}=f(x)+g(x) v+h(x) u, \\
y=0=k(x), \\
z=l(x)+m(x) v,
\end{gathered}
$$

where $x \in M$, with $M$ an open subset of $R^{n}$, $v \in R^{m}, \quad u \in R^{p}, \quad y \in R^{m}, \quad z \in R^{p}$ and $m<n$. Let $g(x)=\left[g_{1}(x), \ldots, g_{m}(x)\right], h(x)=\left[h_{1}(x), \ldots\right.$, $\left.h_{p}(x)\right]$, and $k(x)=\left[k_{1}(x), \ldots, k_{m}(x)\right]^{\mathrm{T}}$, where

\footnotetext{
* Received 17 March 1993; received in final form 4 February 1994. This paper was not presented at any IFAC meeting. This paper was recommended for publication in revised form by Associate Editor Alberto Isidori under the direction of Editor Tamer Bașar. Corresponding author Professor Harris McClamroch. Tel. +1 313763 2355; Fax +1 313763 0578; E-mail mcclamroch@um.cc.umich.edu.

† Department of Mechanical and Production Engineering, National University of Singapore, 10 Kent Ridge Crescent, Singapore 0511 .

¥Professor and Chairman, Department of Aerospace Engineering, University of Michigan, Ann Arbor, MI 48109-2118, U.S.A.
}

$f(x), g_{i}(x), i=1, \ldots, m, h_{i}(x), i=1, \ldots, p$ are smooth vector fields on $M$, and $k(x), l(x)$ and $m(x)$ are smooth mappings on $M$. We subsequently refer to $v$ as the constraint input variables, to $u$ as the control input variables, to $y$ as the constraint output variables, and to $z$ as the control output variables. The constraint input variables $v$ cannot be directly changed and must be viewed as extra variables which appear in the dynamic equations due to the presence of the constraint defined by equation (2). The control input variables $u$ are used to influence the solutions of equations (1)-(3). The variables $y$ denote the outputs of the system which are constrained, and the variables $z$ denote the outputs to be controlled. The control system described by nonlinear differential-algebraic equations (1)-(3) is also referred to as a constrained system. Control systems of the form (1)-(3) represent an important special class among systems referred to as singular systems or implicit differential equations as described in Brenan et al. (1989), Reich (1990) and Rheinboldt (1984).

The class of nonlinear differential-algebraic equations considered in this paper represents a number of physical systems which include mechanical systems with classical holonomic and nonholonomic constraints. Robotic systems with kinematic constraints, which arise in force and position control tasks are often represented by the class of nonlinear differential-algebraic equations studied here (Krishnan, 1992; McClamroch, 1986). Differential-algebraic equations are also known to arise as dynamic models in power systems (Kaprielian et al. 1991; Venkatasubramanian et al., 1990), electrical circuits, interconnected large scale systems, and in quantum mechanics. In general, differential- 
algebraic equations are the basic model of a number of physical systems while state-space models are a simplification.

Simulation of differential-algebraic equations is difficult since they have similar characteristics to stiff differential equations. Most of the research on nonlinear differential-algebraic equations has focused primarily on issues related to numerical integration of such a system of equations (Brenan et al., 1989). Differentialalgebraic models of systems are nonstandard in the sense that they are not state-space models. Well-posedness of the models has to be studied carefully. Analysis of differential-algebraic equations using differential geometric methods is presented in Reich (1990) and Rheinboldt (1984). Based on the theory of differential equations on manifolds, existence and uniqueness results are proved in Rheinboldt (1984) for a class of mixed systems of differential and algebraic equations. The goemetrical interpretation of the relation between differentialalgebraic equations and vector fields on manifolds is further developed in Reich (1990). The literature on feedback control and stability of differential-algebraic equations or implicit differential equations is sparse. Control issues are, in general, more complicated and so is stability theory. Control systems described by nonlinear differential-algebraic equations of the form (1)-(3) are introduced in McClamroch (1990). Existence and uniqueness of solutions, and the feedback stabilization problem for systems described by equations (1)-(3) are considered. The concept of an equivalent state realization for the class of nonlinear differentialalgebraic equations (1)-(3) is developed. Analysis and control design for a class of linear differential-algebraic equations, having the same structure as equations (1)-(3), is developed in detail in Krishnan (1992) and Krishnan and McClamroch (1992). A tracking problem associated with the class of nonlinear differentialalgebraic equations (1)-(3), under the assumption that the reference inputs are sufficiently slowly varying, is considered in Krishnan and McClamroch (1991).

We emphasize that equations (1)-(3) are not a state realization for the constrained system. Consequently, the existence of well-defined solutions of equations (1)-(3) requires justification. In order to guarantee the well-posedness of a control system described by nonlinear differential-algebraic equations (1)-(3), additional assumptions are necessary. Our fundamental assumptions for the class of nonlinear constrained systems defined by equations (1)-(3) are now introduced. Assume there are finite positive integers $r_{1}, \ldots, r_{m}$ such that

(1) if $r_{i} \geq 2$ then $L_{g_{i}} L_{f}^{k} k_{i}(x)=0, \quad \forall k=$ $0, \ldots, r_{i}-2, i, j=1, \ldots, m, \forall x \in M$;

(2) $\operatorname{rank} A(x)=m, \forall x \in M$, where $A(x) \in$ $R^{m \times m}$ whose element on the $i$-th row and $j$-th column is $a_{i j}=L_{g_{i}} L_{f}^{r_{i}-1} k_{i}(x)$;

(3) if $r_{i} \geq 2$ then $L_{h_{i}} L_{f}^{k} k_{i}(x)=0, \quad \forall k=$ $0, \ldots, r_{i}-2, i=1, \ldots, m$.

$$
j=1, \ldots, p, \forall x \in M
$$

The notation $L_{f} k_{i}(x)$ indicates the directional derivative of $k_{i}(x)$ along the vector field $f(x)$; i.e.

$L_{f} k_{i}(x)=\frac{\partial k_{i}(x)}{\partial x} f(x), \quad L_{f}^{k} k_{i}(x)=L_{f}\left(L_{f}^{k-1} k_{i}(x)\right)$

Assumptions (1)-(3) are essentially that the constraint (strong) relative degree vector of $(f, g, k)$ is $\left(r_{1}, \ldots, r_{m}\right)$ and is not greater than the control (strong) relative degree vector of $(f, h, k)$, componentwise (Isidori, 1989). If $r_{i}=r$, $i=1, \ldots, m$, these assumptions are equivalent to the assumption that the index, as defined for nonlinear singular (or implicit) equations as in Brenan et al. (1989), is $r+1$, for all $x \in M$. The following theorem from McClamroch (1990) states that the nonlinear differential-algebraic equations (1)-(3) are well posed.

\section{Theorem 1.1. Define}

$$
\begin{aligned}
N^{*}= & \left\{x \in M \mid L_{f}^{k} k_{i}(x)=0, k=0, \ldots, r_{i}-1,\right. \\
& i=1, \ldots, m\} .
\end{aligned}
$$

Then $N^{*}$ is an $n-\left(r_{1}+\cdots+r_{m}\right)$ dimensional smooth submanifold of $M$. Assume that the initial condition $x(0) \in N^{*}$ and the input function $u:[0, \infty) \rightarrow R^{p}$ is a given integrable function. Then there exists a unique solution $(x(t), v(t))$ (at least locally defined) of the initial value problem corresponding to equations (1)-(3) and the solution satisfies $x(t) \in N^{*}$ for each $t$ for which the solution is defined.

Thus, under the stated assumptions, it is possible to explicitly specify the state-space for the differential-algebraic equations considered. In fact the state-space is the manifold $N^{*}$. Our particular interest is to study feedback systems which are defined using equations (1)-(3) and a feedback map defined by $u: N^{*} \times R^{P} \rightarrow R^{P}$ where

$$
u=\gamma(x)+\sigma(x) w
$$

here $w \in R^{p}$ is the closed loop input. The closed loop constrained system is defined by the 
nonlinear differential-algebraic equations

$$
\begin{gathered}
\dot{x}=\{f(x)+h(x) \gamma(x)\}+g(x) v+h(x) \sigma(x) w, \\
0=k(x), \\
z=l(x)+m(x) v .
\end{gathered}
$$

Assumptions (1)-(3) are satisfied for all closed loop equations of the form (6)-(8). If we assume that the closed loop input $w:[0, \infty) \rightarrow R^{p}$ is a given integrable function and the initial condition data $x(0) \in N^{*}$, then there exists a unique solution (at least locally defined) of the initial value problem corresponding to equations (6)-(8) which satisfies $x(t) \in N^{*}$ for each $t$ for which the solution is defined.

In this paper, we consider the problem of designing a feedback control law of the form (5) so that the control outputs $z(t)$ track desired reference inputs in control systems described by a class of nonlinear differential-algebraic equations (1)-(3) for which Assumptions (1)-(3) hold. Additional assumptions are introduced so that an equivalent state realization of the control system described by nonlinear differentialalgebraic equations (1)-(3) is expressed in a familiar normal form as in Isidori (1989). A nonlinear feedback control law is then proposed which ensures, under appropriate assumptions which are made clear later, that the tracking error in the closed loop system approaches zero exponentially. In particular, applications to simultaneous contact force and position tracking in constrained robot systems are discussed.

\section{FEEDBACK CONTROL DESIGN}

Consider a control system described by nonlinear differential-algebraic equations defined by equations (1)-(3) satisfying Assumptions (1)-(3). Suppose $r(t) \in R^{p}$ is a vector of reference inputs to the differential-algebraic system. In this section, we describe a procedure for designing a nonlinear feedback control law so that the control outputs $z(t)$ track the given reference inputs $r(t)$ and the tracking error in the closed loop system approaches zero exponentially.

Let $f^{*}(x), h^{*}(x), l^{*}(x)$ and $n^{*}(x)$ be defined as

$$
\begin{gathered}
f^{*}(x)=f(x)+g(x) \alpha(x), \\
h^{*}(x)=h(x)+g(x) \beta(x), \\
l^{*}(x)=l(x)+m(x) \alpha(x), \\
n^{*}(x)=m(x) \beta(x),
\end{gathered}
$$

where $\alpha(x)$ and $\beta(x)$ are defined as

$$
\alpha(x)=-A^{-1}(x)\left[\begin{array}{c}
L_{f}^{r_{1}} k_{1}(x) \\
\vdots \\
L_{f}^{r_{m}} k_{m}(x)
\end{array}\right],
$$

$$
\beta(x)=A^{-1}(x)\left[\begin{array}{ccc}
L_{h_{1}} L_{f}^{r_{1}-1} k_{1}(x) & \cdots & L_{h_{p}} L_{f}^{r_{1}-1} k_{1}(x) \\
\vdots & \vdots & \vdots \\
L_{h_{1}} L_{f}^{r_{m}-1} k_{m}(x) & \cdots & L_{h_{p}} L_{f}^{r_{m}-1} k_{m}(x)
\end{array}\right] .
$$

Without loss of generality, the control outputs $z(t)$ can be ordered so that $n^{*}(x)$ is of the form

$$
n^{*}(x)=\left[\begin{array}{c}
O_{s \times p} \\
B_{2}(x)
\end{array}\right]
$$

where $B_{2}(x) \in R^{(p-s) \times p}$ and $0 \leq s \leq p$. Let

and

$$
h^{*}(x)=\left[h_{1}^{*}(x), \ldots, h_{p}^{*}(x)\right],
$$

$$
l^{*}(x)=\left[l_{l}^{*}(x), \ldots, l_{p}^{*}(x)\right]^{\mathrm{T}} .
$$

We assume further that there exist positive integers $\mu_{1}, \mu_{2}, \ldots, \mu_{s}$ such that the following assumptions hold:

(4) if $\mu_{i} \geq 2$ then $L_{h^{*}} L_{f^{*}}^{k} l_{i}^{*}(x)=0$,

$\forall k=0, \ldots, \mu_{i}-2, j=1, \ldots, p$,

$$
i=1, \ldots, s, \forall x \in M
$$

(5) rank $B(x)=p, \forall x \in M$, where $B(x) \in$ $R^{p \times p}$ is defined by

$$
B(x)=\left[\begin{array}{l}
B_{1}(x) \\
B_{2}(x)
\end{array}\right]
$$

and the element in the $i$-th row and $j$-th column of $B_{1}(x) \in R^{\mathrm{s} \times \mathrm{p}}$ is defined by

$$
\begin{array}{r}
{\left[B_{1}(x)\right]_{i, j}=L_{h_{j}^{*}} L_{f^{*}}^{\mu_{i}-1} l_{i}^{*}(x), \quad i=1, \ldots, s,} \\
j=1, \ldots, p .
\end{array}
$$

It is now possible to state the following theorem.

Theorem 2.1. Consider the control system described by nonlinear differential-algebraic equations (1)-(3) satisfying Assumptions (4) and (5) and Assumptions (1)-(3) of Section 1. Let the notation $d k_{i}(x)$ denote the row vector

$$
d k_{i}(x)=\left[\frac{\partial k_{i}(x)}{\partial x_{1}} \cdots \frac{\partial k_{i}(x)}{\partial x_{n}}\right] .
$$

Then the row vectors

$$
\begin{aligned}
& d k_{1}(x), d L_{f} k_{1}(x), \ldots, d L_{f}^{r_{1}-1} k_{1}(x), \\
& \cdot \\
& d k_{m}(x), d L_{f} k_{m}(x), \ldots, d L_{f}^{r_{m}-1} k_{m}(x), \\
& d l_{1}^{*}(x), d L_{f^{*}} l_{1}^{*}(x), \ldots, d L_{f}^{\mu_{1}-1} l_{1}^{*}(x), \\
& \cdot \\
& \cdot \\
& d l_{s}^{*}(x), d L_{f^{*}} l_{s}^{*}(x), \ldots, d L_{f^{*}}^{\mu_{3}-1} l_{s}^{*}(x),
\end{aligned}
$$

are linearly independent for each $x \in M$. 
Proof. Assumptions (1)-(3) imply that the first $\left(r_{1}+r_{2}+\cdots+r_{m}\right)$ row vectors $d k_{1}(x)$ through $d L_{f}^{r_{m}-1}(x)$ are linearly independent for each $x \in M$ (Isidori, 1989). Assumptions (4) and (5) imply that the last $\left(\mu_{1}+\mu_{2}+\cdots+\mu_{s}\right)$ row vectors $d l_{1}^{*}(x)$ through $d L_{f^{*}}^{\mu^{-1}} l_{s}^{*}(x)$ are linearly independent for each $x \in M$ (Isidori, 1989). However, we need to show that the $\left(r_{1}+\cdots+\right.$ $\left.r_{m}+\mu_{1}+\cdots+\mu_{s}\right)$ row vectors $d k_{1}(x)$ through $d L_{f^{*}}^{\mu_{*}-1} l_{s}^{*}(x)$ taken together are linearly independent for each $x \in M$. This is shown by proceeding along the lines of the proof in Isidori (1989, Lemma 5.1.2) with appropriate modifications.

Assume without loss of generality that $r_{1} \geq r_{i}$, $2 \leq i \leq m$, and $\mu_{1} \geq \mu_{i}, 2 \leq i \leq s$. Let $Q$ denote a matrix with rows formed by row vectors $d k_{1}(x)$ through $d L_{f^{*}}^{\mu_{3}-1} l_{s}^{*}(x)$ as defined above and let the matrix $P$ be defined as

$$
\begin{aligned}
P=[ & h_{1}^{*}(x), \ldots, h_{p}^{*}(x), \ldots, a d_{f^{*}}^{\mu_{1}-1} \\
& \times h_{1}^{*}(x), \ldots, a d_{f^{*}}^{\mu_{1}-1} h_{m}^{*}(x), \\
& g_{1}(x), \ldots, g_{m}(x), \ldots, a d_{f}^{r_{1}-1} \\
& \left.\times g_{1}(x), \ldots, a d_{f}^{r_{1}-1} g_{m}(x)\right],
\end{aligned}
$$

where the notation $a d_{f} g$ denotes the Lie bracket $[f, g]$ and $a d_{f g}^{r}=\left[f, a d_{f}^{r-1} g\right]$. Observe that under the stated assumptions

$$
\begin{gathered}
L_{f^{*}}^{k} k_{i}(x)=L_{f}^{k} k_{i}(x), \quad 0 \leq k \leq r_{i}-1, \\
i=1, \ldots, m, \\
L_{f^{*}}^{k} k_{i}(x)=0, \quad k>r_{i}-1, \quad i=1, \ldots, m, \\
L_{h^{*}} L_{f^{*}}^{k} k_{i}(x)=0, \quad k \geq 0, \quad i=1, \ldots, m, \\
j=1, \ldots, p .
\end{gathered}
$$

Using equations (17)-(19) and from Isidori (1989, Lemma 4.1.3) it follows that

$$
\begin{gathered}
\left\langle d L k_{i} k_{i}(x), a d_{f *}^{r} h_{j}^{*}(x)\right\rangle=0, \\
q=0, \ldots, r_{i}-1, \quad j=1, \ldots, m, \\
r=0, \ldots, \mu_{1}-1, \quad j=1, \ldots, p .
\end{gathered}
$$

Moreover for $i=1, \ldots, m, j=1, \ldots, m$ we have

$$
\begin{aligned}
\left\langle d L_{f}^{q} k_{i}(x), a d_{f}^{r} g_{j}(x)\right\rangle & \\
= & \begin{cases}0, & q+r \leq r_{i}-2, \\
(-1)^{r_{i}-1-q} L_{g_{j}} L_{f}^{r_{f}-1} k_{i}(x), & q+r=r_{i}-1 .\end{cases}
\end{aligned}
$$

and for $i=1, \ldots, s, j=1, \ldots, p$ we have

$$
\begin{aligned}
& \left\langle d L_{f^{*}}^{l_{i}^{*}}(x), a d_{f^{*}}^{r} h_{j}^{*}(x)\right\rangle \\
& \quad= \begin{cases}0, & q+r \leq \mu_{i}-2, \\
(-1)^{\mu_{i}-1-q} L_{h_{j}^{*}} L_{f^{*}}^{\mu_{i}-1} l_{i}^{*}(x), & q+r=\mu_{i}-1 .\end{cases}
\end{aligned}
$$

Thus, the matrix $Q P$ after reordering of rows is of the form

$$
\left[\begin{array}{cc}
0 & A_{1} \\
A_{2} & A_{2}
\end{array}\right]
$$

where $A_{1}$ and $A_{2}$ are matrices having a block triangular structure. The diagonal blocks of $A_{1}$ consist of rows of the matrix $A(x)$ defined by Assumption (2) of Section 1. The diagonal blocks of $A_{2}$ consist of rows of the matrix $B_{1}(x)$ defined by Assumption (5). Thus the row vectors defined above are linearly independent for each $x \in M$.

We now consider new variables defined by

$$
\left(\begin{array}{c}
\tilde{x} \\
\bar{x} \\
\xi
\end{array}\right)=\left(\begin{array}{l}
\Psi_{0}(x) \\
\Psi_{1}(x) \\
\Psi_{2}(x)
\end{array}\right)=\Phi(x),
$$

where

$$
\begin{aligned}
& \begin{array}{l}
\tilde{x}_{1}=k_{1}(x), \quad \tilde{x}_{2}=L_{f} k_{1}(x), \ldots, \tilde{x}_{r_{1}}=L_{f}^{r_{1}-1} k_{1}(x), \\
\tilde{x}_{r_{1}+1}=k_{2}(x), \quad \tilde{x}_{r_{1}+2}=L_{f} k_{2}(x) \\
, \ldots, \tilde{x}_{r_{1}+\cdots+r_{m}}=L_{f}^{r_{m}-1} k_{m}(x), \\
\bar{x}_{1}=l_{1}^{*}(x), \quad \bar{x}_{2}=L_{f^{*}} l_{1}^{*}(x), \ldots, \bar{x}_{\mu_{1}}=L_{f^{*}}^{\mu_{1}-1} l_{1}^{*}(x), \\
\bar{x}_{\mu_{1}+1}=l_{2}^{*}(x), \quad \bar{x}_{\mu_{1}+2}=L_{f^{*}} l_{2}^{*}(x) \\
\text { and } \quad, \ldots, \bar{x}_{\mu_{1}+\cdots+\mu_{s}}=L_{f^{*}}^{\mu_{3}-1} l_{s}^{*}(x), \\
\xi \xi=\Phi_{2}(x),
\end{array}
\end{aligned}
$$

where the vector $\xi \in R^{n-\left(r_{1}+\cdots+r_{m}\right)-\left(\mu_{1}+\cdots+\mu_{1}\right)}$ and $\Psi_{2}(\cdot)$ is a smooth mapping. Theorem 2.1 guarantees that there exists a nonlinear coordinate change defined by equations (20)-(22) which is a local diffeomorphism in a neighborhood of every $x \in M$. Clearly, Theorem 2.1 is only necessary for equations (20)-(22) to be a global diffeomorphism on $M$. Sufficient conditions which guarantee the global validity of such a diffeomorphism on $M$ can be given as in Byrnes and Isidori (1991). However, the conditions would be very restrictive. Here we assume that equations (20)-(22) define a diffeomorphism on $M$. The differential-algebra equations (1)-(3) in the transformed coordinates are given by

$$
\begin{aligned}
& \dot{\tilde{x}}_{1}=\tilde{x}_{2} \\
& \cdot \\
& \dot{\tilde{x}}_{r_{1}-1}=\tilde{x}_{r_{1}} \\
& \dot{\tilde{x}}_{r_{1}}=L_{f}^{r_{1}} k_{1}(x)+\sum_{j=1}^{m}\left(L_{f_{j}} L_{f}^{r_{1}-1} k_{1}(x)\right) v_{j} \\
& \quad+\sum_{j=1}^{p}\left(L_{h_{j}} L_{f}^{r_{1}-1} k_{1}(x)\right) u_{j}
\end{aligned}
$$




$$
\begin{aligned}
& \dot{\tilde{x}}_{r_{1}+\cdots+r_{m-1}+1}=\tilde{x}_{r_{1}+\cdots+r_{m-1}+2} \\
& \dot{\tilde{x}}_{r_{1}+\cdots+r_{m}-1}=\tilde{x}_{r_{1}+\cdots+r_{m}} \\
& \dot{\tilde{x}}_{r_{1}+\cdots+r_{m}}=L_{f}^{r_{m}} k_{m}(x)+\sum_{j=1}^{m}\left(L_{g_{j}} L_{f}^{r_{m}-1} k_{m}(x)\right) v_{j} \\
& \quad+\sum_{j=1}^{p}\left(L_{h_{j}} L_{f}^{r_{m}-1} k_{m}(x)\right) u_{j} \\
& \dot{x}=\frac{\partial \Psi_{1}(x)}{\partial x}(f(x)+g(x) v+h(x) u) \\
& \dot{\xi}=\frac{\partial \Psi_{2}(x)}{\partial x}(f(x)+g(x) v+h(x) u) \\
& y_{i}=0=\tilde{x}_{r_{i-1}+1}, \quad i=1, \ldots, m, \quad r_{0}=0 \\
& z=l(x)+m(x) v,
\end{aligned}
$$

where

$$
x=\Phi^{-1}\left(\begin{array}{c}
\tilde{x} \\
\bar{x} \\
\xi
\end{array}\right) .
$$

Clearly, eliminating the constraints in the transformed variables amounts to setting the variables $\tilde{x}$ identically equal to zero. Setting $\tilde{x}=0$ in equation (23) we obtain

$$
v=\alpha(x)+\beta(x) u,
$$

where $\alpha(x)$ and $\beta(x)$ are defined by equations (13) and (14). Moreover the nonlinear differential-algebraic equations are equivalent to the $n-\left(r_{1}+r_{2}+\cdots r_{m}\right)$ dimensional nonlinear state realization given by

$$
\begin{aligned}
& \dot{x}_{1}=\bar{x}_{2} \\
& \cdot \\
& \dot{x}_{\mu_{1}-1}=\bar{x}_{\mu_{1}} \\
& \dot{x}_{\mu_{1}}=L_{f^{*}}^{\mu_{1}} l_{1}^{*}(x)+\sum_{j=1}^{p}\left(L_{h_{j}^{*}} L_{f^{*}}^{\mu_{1}-1} l_{1}^{*}(x)\right) u_{j} \\
& \cdot \\
& \dot{\bar{x}}_{\mu_{1}+\cdots+\mu_{s-1}+1}=\bar{x}_{\mu_{1}+\cdots+\mu_{s-1}+2} \\
& \cdot \\
& \dot{x}_{\mu_{1}+\cdots+\mu_{s}-1}=\bar{x}_{\mu_{1}+\cdots+\mu_{s}} \\
& \dot{x}_{\mu_{1}+\cdots+\mu_{s}}=L_{f}^{\mu_{s}} l_{s}^{*}(x)+\sum_{j=1}^{p}\left(L_{h_{j}^{*}} L_{f^{*}}^{\mu_{s}-1} l_{s}^{*}(x)\right) u_{j} \\
& \dot{\xi}=\frac{\partial \Psi_{2}(x)}{\partial x} f^{*}(x)+\frac{\partial \Psi_{2}(x)}{\partial x} h^{*}(x) u \\
& z=l^{*}(x)+\left[\begin{array}{c}
0_{s \times p} \\
B_{2}(x)
\end{array}\right] u, \\
&
\end{aligned}
$$

where

$$
x=\Phi^{-1}\left(\begin{array}{c}
0 \\
\bar{x} \\
\xi
\end{array}\right) \text {. }
$$

The equivalent state realizations for the nonlinear differential-algebraic equations are in a familiar normal form as described in Isidori (1989). Suppose the control outputs $z(t)$ are required to track the reference inputs $r(t)$; i.e. we want

$$
z(t) \rightarrow r(t) \text { as } t \rightarrow \infty .
$$

Then a nonlinear feedback control law can be chosen as

$$
u(x, R)=\left[\begin{array}{l}
B_{1}(x) \\
B_{2}(x)
\end{array}\right]^{-1}\left\{-\left[\begin{array}{l}
a_{1}(x) \\
a_{2}(x)
\end{array}\right]+\bar{u}\right\},
$$

where

$$
\begin{gathered}
R(t)=\left(r_{1}(t), \ldots, r_{1}^{\mu_{1}-1}(t), \ldots, r_{s}(t), \ldots, r_{s}^{\mu_{s}-1}(t),\right. \\
\left.r_{(s+1)}(t), \ldots, r_{p}(t)\right)^{\mathrm{T}}, \\
\bar{u}_{i}=r_{i}^{\mu_{i}}(t)-\sum_{j=1}^{\mu_{i}} K_{i, j}\left(L_{f^{*}}^{j-1} l_{i}^{*}(x)-r_{i}^{j-1}(t)\right), \\
i=1, \ldots, s, \\
\bar{u}_{i}=r_{i}(t), \quad i=s+1, \ldots, p, \\
a_{1}(x)=\left[\begin{array}{c}
L_{f^{*}}^{\mu_{1}} l_{1}^{*}(x) \\
: \\
L_{f}^{\mu_{j}} l_{s}^{*}(x)
\end{array}\right], \quad a_{2}(x)=\left[\begin{array}{c}
l_{s+1}^{*}(x) \\
: \\
l_{p}^{*}(x)
\end{array}\right],
\end{gathered}
$$

and $\gamma^{j}(t)$ denotes the $j$-th time derivative of $\gamma(t)$. Using such a control law the tracking errors in the closed loop differential-algebraic system defined by

$$
e_{i}(t)=z_{i}(t)-r_{i}(t), \quad i=1, \ldots, p,
$$

satisfy the following equations:

$$
\begin{gathered}
e_{i}^{\mu_{i}}(t)+K_{i, \mu_{i}} e_{i}^{\mu_{i}-1}(t)+\cdots+K_{i, 1} e_{i}(t)=0, \\
i=1, \ldots, s, \\
e_{i}(t)=0, \quad i=s+1, \ldots, p .
\end{gathered}
$$

If the gains $K_{i, j}$ are chosen such that the polynomials

$$
s^{\mu_{i}}+K_{i, \mu_{i}} s^{\mu_{i}-1}+\cdots+K_{i, 1}=0, \quad i=1, \ldots, s,
$$

are Hurwitz then the tracking error for the closed loop differential-algebraic system for the control outputs $z_{i}(t), \quad i=1, \ldots, s$ approaches zero exponentially; i.e.

$e_{i}(t)=z_{i}(t)-r_{i}(t) \rightarrow 0 \quad$ as $\quad t \rightarrow \infty, \quad i=1, \ldots, s$.

The tracking error for the control outputs $z_{i}(t)$, $i=s+1, \ldots, p$ is identically equal to zero at all 
times; i.e.

$$
e_{i}(t)=0, \quad \forall t \geq 0, \quad i=s+1, \ldots, p .
$$

Additionally, in order to guarantee the boundedness of all the internal states and the control inputs in the closed loop system the following assumption is necessary.

Assumption 2.2. The subsystem

$$
\dot{\xi}=\frac{\partial \Psi_{2}(x)}{\partial x} f^{*}(x)+\frac{\partial \Psi_{2}(x)}{\partial x} h^{*}(x) u(x, R),
$$

where

$$
x=\Phi^{-1}\left(\begin{array}{l}
0 \\
\bar{x} \\
\xi
\end{array}\right)
$$

is bounded input bounded state stable with $\bar{x}, R$ as inputs.

If Assumption 2.2 is satisfied then the control inputs and all the internal states of the closed loop differential-algebraic system are bounded. This does not depend on the choice of the mapping $\Psi_{2}(x)$ in equation (20). Sufficient conditions which guarantee Assumption 2.2 can be given as in Sastry and Isidori (1989). They are as follows:

(i) the subsystem defined by equation (35) with $\bar{x}(t), R(t)$ set identically equal to zero is globally exponentially stable; and

(ii) the vector field defined by the right-hand side of equation (35) is globally Lipschitz.

In this section, a procedure has been presented for the design of a nonlinear feedback control law in order to achieve tracking of reference inputs in control systems described by nonlinear differential-algebraic equations of the form (1)-(3). The feedback control law ensures that the tracking error in the closed loop differential-algebraic system approaches zero exponentially. The methodology presented here is used in the Section 3 to design feedback control laws for simultaneous force and position tracking in constrained robot systems.

3. SIMULTANEOUS FORCE AND POSITION TRACKING IN CONSTRAINED ROBOT SYSTEMS

Robotic systems often operate in a constrained environment and interact with these environmental constraints to perform certain tasks. Examples of such challenging tasks include parts insertion and assembly, crank turning, polishing, grinding, deburring, contour following, writing, etc. These tasks may involve a single robot or multiple robots working together. Thus, constrained robot systems and more generally constrained mechanical systems represent a class of dynamical systems of both practical and theoretical importance. Constraint forces, which maintain satisfaction of the constraints, are important aspects of these systems. Successful accomplishment of constrained tasks requires simultaneous force and position control. Constrained robotic systems are often described by differential-algebraic equations of the form (1)-(33). In this section, we consider the problem of designing feedback control laws that achieve simultaneous force and position tracking in constrained robot systems. The control laws are designed based on the theory presented in Section 2.

\subsection{Constrained robot systems with rigid joints}

We consider mathematical representations which model a class of constrained robot systems. Examples of such systems include: a robot with end effector in contact with a rigid surface where the contact force of the end effector on the surface and the position of the end effector on the surface needs to be controlled simultaneously; two robots handling a common object where the motion of the object and the squeeze force or the interaction force exerted on the object by the robots needs to be controlled simultaneously; closed chain manipulators; walking robots; grasping; and coordinated motion of an object using multifingered robot hands, etc. The constraints are modeled as holonomic constraints. Contact forces or constraint forces, which are produced by the interaction of the robot system with the constraint surface, are important aspects of these systems and successful accomplishment of constrained tasks requires contact force control in addition to position control on the surface. The joints of the robotic manipulator are assumed to be actuated by direct drive actuators and it is also assumed that the robot joints are driven by rigid mechanisms, i.e. there is no joint flexibility. The model of the constrained robot system, under such an assumption, is represented as Krishnan (1992), McClamroch (1986) and McClamroch and Wang (1988)

$$
\begin{gathered}
M(q) \ddot{q}+H(q, \dot{q})=J^{\mathrm{T}}(q) \lambda+u, \\
y=0=\phi(q), \\
z_{1}=h_{1}(q), \\
z_{2}=\lambda,
\end{gathered}
$$

where $q \in R^{n}$ denotes the vector of generalized displacements, $M(q)$ is the inertia matrix which is symmetric and positive definite, $H(q, \dot{q})$ denotes the vector of nonlinearities which include the Coriolis, centripetal and gravity forces, $u \in R^{n}$ represents the vector of generalized 
forces applied at each joint, $\lambda \in R^{m}$ is a vector of multipliers corresponding to the constraint vector function $\phi(\cdot): R^{n} \rightarrow R^{m}$, and

$$
J(q)=\frac{\partial \phi(q)}{\partial q}
$$

is the Jacobian matrix of $\phi(q)$ which is assumed to have full row rank. The control outputs represented by the vector $z_{1} \in R^{n-m}$ are chosen such that together with the constraints $\phi(q)=0$, they specify completely the position vector $q$. The control outputs represented by the vector $z_{2} \in R^{m}$ are chosen to be the constraint forces $\lambda$. For this model, the constraint manifold $N^{*}$ defined by equation (4) is given by

$$
N^{*}=\{(q, \dot{q}): \phi(q)=0, J(q) \dot{q}=0\} .
$$

Simultaneous force and position control for constrained robot systems modeled by equations (37)-(40) has been an active area of recent research (see Cai and Goldenberg, 1989; Chen and Shayman, 1992; Cole, 1989; Jankowski and Elmaraghy, 1992; Krishnan, 1992; Krishnan and McClamroch, 1990; Liu, 1991; McClamroch and Wang, 1988; Mills, 1992; Mills and Goldenberg, 1989; Ramadorai et al., 1992; Wen and Delgado, 1992; Yoshikawa, 1987; Yun, 1988 and the references therein). A number of approaches have been proposed for designing control laws which achieve local regulation of contact force and position vectors, and tracking of contact force and position trajectories. In this section, we design a feedback control law for tracking desired force and position trajectories based on the development in Section 2. The design is, in principle, similar to the control law in McClamroch and Wang (1988) when the constraint outputs $y$ defined by equation (38) take the form

$$
y=0=q_{1}-\Omega\left(q_{2}\right),
$$

and the control outputs $z_{1}$ defined by equation (39) take the form

$$
z_{1}=h_{1}(q)=q_{2},
$$

where $q_{1} \in R^{m}$ and $q_{2} \in R^{n-m}$.

Suppose $r_{1}(t)$ and $r_{2}(t)$ are vectors of desired position and force trajectories. Our goal is to design a feedback control law so that the position outputs $z_{1}(t)$ and the force outputs $z_{2}(t)$ for the constrained robot system track the desired position and force trajectories $r_{1}(t)$ and $r_{2}(t)$, respectively. We use the development in Section 2 to design a feedback control law so that the closed loop system has the desired force and position tracking properties. Equations (37)-(40) can be rewritten in a first-order form as

$$
\begin{gathered}
\left(\begin{array}{c}
\dot{q} \\
\ddot{q}
\end{array}\right)=f(q, \dot{q})+g(q, \dot{q}) \lambda+h(q, \dot{q}) u \\
y=0=k(q, \dot{q}) \\
z=l(q, \dot{q})+m(q, \dot{q}) \lambda
\end{gathered}
$$

where

$$
\begin{gathered}
f(q, \dot{q})=\left[\begin{array}{c}
\dot{q} \\
-M^{-1}(q) H(q, \dot{q})
\end{array}\right], \\
g(q, \dot{q})=\left[\begin{array}{c}
0 \\
M^{-1}(q) J^{\mathrm{T}}(q)
\end{array}\right], \\
h(q, \dot{q})=\left[\begin{array}{c}
0 \\
M^{-1}(q)
\end{array}\right], \quad k(q, \dot{q})=\phi(q), \\
l(q, \dot{q})=\left[\begin{array}{c}
h_{1}(q) \\
0
\end{array}\right], \quad m(q, \dot{q})=\left[\begin{array}{c}
0 \\
I_{m}
\end{array}\right] .
\end{gathered}
$$

The differential-algebraic equations (43)-(45) satisfy Assumptions (1)-(3) of Section 1 with a constraint relative degree vector $\left(r_{1}, r_{2}, \ldots, r_{m}\right)$ $=(2,2, \ldots, 2)$ and control relative degree vector $(2,2, \ldots, 2)$. From equations (9)-(12), $f^{*}(q, \dot{q})$, $h^{*}(q, \dot{q}), \quad l^{*}(q, \dot{q})$, and $n^{*}(q, \dot{q})$ for the constrained robot model represented by equations (37) $-(40)$ are given by

$$
\begin{gathered}
f^{*}(q, \dot{q})=\left[\begin{array}{c}
\dot{q} \\
-M^{-1}(q) H(q, \dot{q})
\end{array}\right] \\
+\left[\begin{array}{c}
0 \\
M^{-1}(q) J^{\mathrm{T}}(q)\left(J(q) M^{-1}(q) J^{\mathrm{T}}(q)\right)^{-1} \\
\times\left[J(q) M^{-1}(q) H(q, \dot{q})-j(q) \dot{q}\right]
\end{array}\right], \\
h^{*}(q, \dot{q})=\left[\begin{array}{c}
0 \\
M^{-1}(q)\left\{I-J^{\mathrm{T}}(q)\left(J(q) M^{-1}\right.\right. \\
\left.\left.\times(q) J^{\mathrm{T}}(q)\right)^{-1} J(q) M^{-1}(q)\right\}
\end{array}\right], \\
l^{*}(q, \dot{q})=\left[\begin{array}{c}
\left(J(q) M^{-1}(q) J^{\mathrm{T}}(q)\right)^{-1}[J(q) \\
\left.\times M^{-1}(q) H(q, \dot{q})-j(q) \dot{q}\right]
\end{array}\right],
\end{gathered}
$$

$n^{*}(q, \dot{q})=\left[\begin{array}{c}0 \\ -\left(J(q) M^{-1}(q) J^{\mathrm{T}}(q)\right)^{-1} J(q) M^{-1}(q)\end{array}\right]$.

Assumptions (4) and (5) of Section 2 are satisfied in this case with $\left(\mu_{1}, \mu_{2}, \ldots, \mu_{n-m}\right)=$ $(2,2, \ldots, 2)$. Now the coordinate change defined by equations $(20)-(22)$ in this case is given by

$$
\begin{gathered}
\tilde{x}_{1}=\phi(q), \\
\tilde{x}_{2}=J(q) \dot{q}, \\
\bar{x}_{1}=h_{1}(q), \\
\bar{x}_{2}=\frac{\partial h_{1}(q)}{\partial q} \dot{q}=P(q) \dot{q} .
\end{gathered}
$$


Following equations (23)-(27), an equivalent state realization is obtained for the differentialalgebraic equations (37)-(40) which is given by

$$
\begin{aligned}
\dot{x}_{1}=\bar{x}_{2} & \\
\dot{x}_{2}= & \dot{P}(q) \dot{q}-P(q) M^{-1}(q)\left\{H(q, \dot{q})-J^{\mathrm{T}}(q)\right. \\
& \times\left(J(q) M^{-1}(q) J^{\mathrm{T}}(q)\right)^{-1} \\
& \left.\times\left[J(q) M^{-1}(q) H(q, \dot{q})-\dot{J}(q) \dot{q}\right]\right\} \\
& +P(q) M^{-1}(q)\left\{I-J^{\mathrm{T}}(q)\left(J(q) M^{-1}(q) J^{\mathrm{T}}(q)\right)^{-1}\right. \\
& \left.\times J(q) M^{-1}(q)\right\} u, \\
& \\
z_{2}= & \left(J(q) M^{-1}(q) J^{\mathrm{T}}(q)\right)^{-1}\left[J(q) M^{-1}(q) H(q,\right.
\end{aligned}
$$

where from equations $(50)-(53)$ we have

$$
q=\psi\left(\bar{x}_{1}\right), \quad \dot{q}=Q(q) \bar{x}_{2} .
$$

The nonlinear feedback control law defined by equation (28) is now given by

$$
\begin{aligned}
u(t)= & M(q) Q(q)\left(\ddot{r}_{1}-\Lambda_{1}\left(h_{1}(q)-r_{1}\right)\right. \\
& \left.-\Lambda_{2}\left(P(q) \dot{q}-\dot{r}_{1}\right)\right)+H(q, \dot{q}) \\
& +M(q) \dot{Q}(q) P(q) \dot{q}-J^{\mathrm{T}}(q) r_{2} \\
& +J^{\mathrm{T}}(q) \bar{G}\left(\lambda-r_{2}\right),
\end{aligned}
$$

where $\bar{G} \in R^{m \times m}$ is a positive semidefinite matrix and $\Lambda_{1}$ and $\Lambda_{2}$ are positive definite matrices. The tracking errors in the closed loop constrained system satisfy the equations

$$
\begin{gathered}
\ddot{e}_{1}(t)+\Lambda_{2} \dot{e}_{1}(t)+\Lambda_{1} e_{1}(t)=0, \\
\left(J(q) M^{-1}(q) J^{\mathrm{T}}(q)\right)\left(I_{m}+\bar{G}\right) e_{2}(t)=0,
\end{gathered}
$$

where the position tracking error is defined as

$$
e_{1}(t)=h_{1}(q)-r_{1}(t)
$$

and the force tracking error is defined as

$$
e_{2}(t)=\lambda(t)-r_{2}(t) .
$$

Since $\Lambda_{1}$ and $\Lambda_{2}$ are chosen as positive definite matrices, the tracking error $e_{1}(t)$ for the position outputs approach zero exponentially and the force tracking error $e_{2}(t)$ is identically zero at all times. In addition, all the internal states of the constrained robot system and the control inputs remain bounded since Assumption 2.2 of Section 2 is satisfied trivially. The control law requires feedback of position and velocity variables. The control law (59) matches the control law (28) when $\bar{G}$ is a zero matrix. However, when $\bar{G}$ is not a zero matrix, force feedback can also be included even though it is not essential for achieving the tracking objective.

\subsection{Constrained robot systems with flexible joints}

The model of a constrained robot system with holonomic constraints, described by equations
(38)-(40), is derived under the assumption that the joints of the robot system are actuated by direct drive mechanisms which are rigid. Often joints of a mechanical system exhibit some elasticity due to the drive mechanism, e.g. harmonic drives, gears, etc. This leads to low frequency resonance in the response of the system. In this section, we consider mathematical representations which model a class of constrained robot systems which include the effects of joint flexibility. The model, obtained by modifying equations (37)-(40) to include effects of joint flexibility as in Spong (1987), is represented as

$$
M\left(q_{1}\right) \ddot{q}_{1}+H\left(q_{1}, \dot{q}_{1}\right)=J^{\mathrm{T}}\left(q_{1}\right) \lambda+K_{2}\left(q_{2}-q_{1}\right),
$$

$$
\begin{gathered}
M_{2} \ddot{q}_{2}+K_{2}\left(q_{2}-q_{1}\right)=u, \\
y=0=\phi\left(q_{1}\right), \\
z_{1}=h_{1}\left(q_{1}\right), \\
z_{2}=\lambda,
\end{gathered}
$$

where $q_{1} \in R^{n}$ denotes the vector of generalized displacements of the links of the robot, $q_{2} \in R^{n}$ denotes the vector of generalized displacements of the actuator shafts, $M_{2}$ is a constant positive definite matrix which represents the actuator inertia and $k_{2}$ is a constant positive definite matrix representing the stiffness coefficients of the joints of the robot. All the other variables have the same description as in equations (37)-(42) with $q$ replaced by $q_{1}$. Approaches to simultaneous contact force and position control in constrained robot systems with joint flexibility have been proposed in Ahmad (1991), Jankowski and Elmaraghy (1992), Krishnan and McClamroch (1990) and Mills (1992). In this section, we use the development in Section 2 for designing a feedback control law in order to achieve tracking of contact force and position in constrained robot systems with joint flexibility.

Suppose $r_{1}(t)$ and $r_{2}(t)$ are vectors of desired position and force trajectories. Equations (64)(68) can be rewritten in a first-order form as

$$
\begin{gathered}
\left(\begin{array}{c}
\dot{q}_{1} \\
\ddot{q}_{1} \\
\dot{q}_{2} \\
\ddot{q}_{2}
\end{array}\right)= \\
\quad f\left(q_{1}, \dot{q}_{1}, q_{2}, \dot{q}_{2}\right)+g\left(q_{1}, \dot{q}_{1}, q_{2}, \dot{q}_{2}\right) \lambda \\
+h\left(q_{1}, \dot{q}_{1}, q_{2}, \dot{q}_{2}\right) u, \\
\quad y=0=k\left(q_{1}, \dot{q}_{1}, q_{2}, \dot{q}_{2}\right), \\
z=l\left(q_{1}, \dot{q}_{1}, q_{2}, \dot{q}_{2}\right)+m\left(q_{1}, \dot{q}_{1}, q_{2}, \dot{q}_{2}\right) \lambda,
\end{gathered}
$$


where

$$
\begin{gathered}
f\left(q_{1}, \dot{q}_{1}, q_{2}, \dot{q}_{2}\right) \\
=\left[\begin{array}{c}
\dot{q}_{1} \\
M^{-1}\left(q_{1}\right)\left[-H\left(q_{1}, \dot{q}_{1}\right)+K_{2}\left(q_{2}-q_{1}\right)\right] \\
\dot{q}_{2} \\
-M_{2}^{-1} K_{2}\left(q_{2}-q_{1}\right)
\end{array}\right], \\
g\left(q_{1}, \dot{q}_{1}, q_{2}, \dot{q}_{2}\right)=\left[\begin{array}{c}
0 \\
M^{-1}\left(q_{1}\right) J^{\mathrm{T}}\left(q_{1}\right) \\
0 \\
0
\end{array}\right], \\
h\left(q_{1}, \dot{q}_{1}, q_{2}, \dot{q}_{2}\right)=\left[\begin{array}{c}
0 \\
0 \\
0 \\
M_{2}^{-1}
\end{array}\right], \\
k\left(q_{1}, \dot{q}_{1}, q_{2}, \dot{q}_{2}\right)=\phi\left(q_{1}\right), \\
l\left(q_{1}, \dot{q}_{1}, q_{2}, \dot{q}_{2}\right)=\left[\begin{array}{c}
h_{1}\left(q_{1}\right) \\
0
\end{array}\right], \\
m\left(q_{1}, \dot{q}_{1}, q_{2}, \dot{q}_{2}\right)=\left[\begin{array}{c}
0 \\
I_{m}
\end{array}\right] .
\end{gathered}
$$

These differential-algebraic equations satisfy Assumptions (1)-(3) of Section 1 with a constraint relative degree vector $\left(r_{1}, r_{2}, \ldots, r_{m}\right)$ $=(2,2, \ldots, 2)$ and control relative degree vector $(4,4, \ldots, 4)$. Define

$$
\begin{aligned}
\alpha\left(q_{1}, \dot{q}_{1}, q_{2}\right)= & \left(J\left(q_{1}\right) M^{-1}\left(q_{1}\right) J^{\mathrm{T}}\left(q_{1}\right)\right)^{-1} \\
& \times\left[J\left(q_{1}\right) M^{-1}\left(q_{1}\right) H\left(q_{1}, \dot{q}_{1}\right)\right. \\
& -\dot{J}\left(q_{1}\right) \dot{q}_{1}-J\left(q_{1}\right) M^{-1}\left(q_{1}\right) \\
& \left.\times K_{2}\left(q_{2}-q_{1}\right)\right] .
\end{aligned}
$$

From equations (9)-(12), $f^{*}, h^{*}, l^{*}$, and $n^{*}$ are given by

$$
\begin{aligned}
& f^{*}\left(q_{1}, \dot{q}_{1}, q_{2}, \dot{q}_{2}\right) \\
& =f\left(q_{1}, \dot{q}_{1}, q_{2}, \dot{q}_{2}\right)+g\left(q_{1}, \dot{q}_{1}, q_{2}, \dot{q}_{2}\right) \\
& \times \alpha\left(q_{1}, \dot{q}_{1}, q_{2}, \dot{q}_{2}\right), \\
& n^{*}\left(q_{1}, \dot{q}_{1}, q_{2}, \dot{q}_{2}\right)=0, \\
& h^{*}\left(q_{1}, \dot{q}_{1}, q_{2}, \dot{q}_{2}\right)=h\left(q_{1}, \dot{q}_{1}, q_{2}, \dot{q}_{2}\right), \\
& l^{*}\left(q_{1}, \dot{q}_{1}, q_{2}, \dot{q}_{2}\right)=\left[\begin{array}{c}
h_{1}\left(q_{1}\right) \\
\alpha\left(q_{1}, \dot{q}_{1}, q_{2}, \dot{q}_{2}\right)
\end{array}\right] .
\end{aligned}
$$

Assumptions (4) and (5) of Section 2 are satisfied in this case with $\left(\mu_{1}, \ldots, \mu_{n-m}, \mu_{n-m+1}\right.$, $\left.\ldots, \mu_{n}\right)=(4, \ldots, 4,2, \ldots, 2)$. Now the coordinate change defined by equations (20)-(22) in this case is given by

$$
\begin{gathered}
\tilde{x}_{1}=\phi\left(q_{1}\right), \\
\tilde{x}_{2}=J\left(q_{1}\right) \dot{q}_{1}, \\
\bar{x}_{1}=h_{1}\left(q_{1}\right),
\end{gathered}
$$

$$
\bar{x}_{2}=\frac{\partial h_{1}\left(q_{1}\right)}{\partial q_{1}} \dot{q}_{1}=P\left(q_{1}\right) \dot{q}_{1},
$$

$$
\begin{gathered}
\bar{x}_{3}=L_{f^{*}}^{2} h_{1}\left(q_{1}\right)=f_{1}\left(q_{1}, \dot{q}_{1}\right)+B_{1}\left(q_{1}\right) q_{2}, \\
\bar{x}_{4}=L_{f^{*}}^{3} h_{1}\left(q_{1}\right)=f_{2}\left(q_{1}, \dot{q}_{1}, q_{2}\right)+B_{1}\left(q_{1}\right) \dot{q}_{2}, \\
\bar{x}_{5}=\alpha\left(q_{1}, \dot{q}_{1}, q_{2}\right)=f_{3}\left(q_{1}, \dot{q}_{1}\right)+B_{2}\left(q_{1}\right) q_{2}, \\
\bar{x}_{6}=L_{f^{*}} \alpha\left(q_{1}, \dot{q}_{1}, q_{2}\right)=f_{4}\left(q_{1}, \dot{q}_{1}, q_{2}\right)+B_{2}\left(q_{1}\right) \dot{q}_{2},
\end{gathered}
$$

where $f_{1}, B_{1}, f_{2}, B_{2}, f_{3}, f_{4}$ are defined in the Appendix. Following equations (23)-(27), an equivalent state realization is obtained for the differential-algebraic equations which is given by

$$
\begin{gathered}
\dot{x}_{1}=\bar{x}_{2}, \\
\dot{x}_{2}=\bar{x}_{3}, \\
\dot{x}_{3}=\bar{x}_{4}, \\
\dot{x}_{4}=A_{1}\left(q_{1}, \dot{q}_{1}, q_{2}, \dot{q}_{2}\right)+B_{1}\left(q_{1}\right) M_{2}^{-1} u, \\
\dot{x}_{5}=\bar{x}_{6}, \\
\dot{x}_{6}=A_{2}\left(q_{1}, \dot{q}_{1}, q_{2}, \dot{q}_{2}\right)+B_{2}\left(q_{1}\right) M_{2}^{-1} u, \\
z_{1}=\bar{x}_{1}, \\
z_{2}=\bar{x}_{5},
\end{gathered}
$$

where

$$
\begin{gathered}
q_{1}=\psi\left(\bar{x}_{1}\right), \quad \dot{q}_{1}=Q\left(q_{1}\right) \bar{x}_{2}, \\
q_{2}=\left[\begin{array}{l}
B_{1}\left(q_{1}\right) \\
B_{2}\left(q_{2}\right)
\end{array}\right]^{-1}\left\{-\left(\begin{array}{l}
f_{1}\left(q_{1}, \dot{q}_{1}\right) \\
f_{3}\left(q_{1}, \dot{q}_{1}\right)
\end{array}\right)+\left(\begin{array}{l}
\bar{x}_{3} \\
\bar{x}_{5}
\end{array}\right)\right\}, \\
\dot{q}_{2}=\left[\begin{array}{l}
B_{1}\left(q_{1}\right) \\
B_{2}\left(q_{2}\right)
\end{array}\right]^{-1}\left\{-\left(\begin{array}{l}
f_{2}\left(q_{1}, \dot{q}_{1}, q_{2}\right) \\
f_{4}\left(q_{1}, \dot{q}_{1}, q_{2}\right)
\end{array}\right)+\left(\begin{array}{l}
\bar{x}_{4} \\
\bar{x}_{6}
\end{array}\right)\right\}
\end{gathered}
$$

and $A_{1}$ and $A_{2}$ are defined in the Appendix. The nonlinear feedback control law defined by equation (28) is now given by

$$
\begin{aligned}
u(t)= & M_{2}\left[\begin{array}{l}
B_{1}\left(q_{1}\right) \\
B_{2}\left(q_{1}\right)
\end{array}\right]^{-1}\left\{-\left(\begin{array}{l}
A_{1}\left(q_{1}, \dot{q}_{1}, q_{2}, \dot{q}_{2}\right) \\
A_{2}\left(q_{1}, \dot{q}_{1}, q_{2}, \dot{q}_{2}\right)
\end{array}\right)\right. \\
& \left.+\left(\begin{array}{l}
v_{1}(r) \\
v_{2}(t)
\end{array}\right)\right\},
\end{aligned}
$$

where

$$
\begin{aligned}
v_{1}(t) & =r_{1}^{(4)}(t)-\Lambda_{1}\left(h_{1}\left(q_{1}\right)-r_{1}(t)\right) \\
& -\Lambda_{2}\left(P\left(q_{1}\right) \dot{q}_{1}-r_{1}^{(1)}(t)\right) \\
& -\Lambda_{3}\left(f_{1}\left(q_{1}, \dot{q}_{1}\right)+B_{1}\left(q_{1}\right) q_{2}-r_{1}^{(2)}(t)\right) \\
& -\Lambda_{4}\left(f_{2}\left(q_{1}, \dot{q}_{1}, q_{2}\right)+B_{1}\left(q_{1}\right) \dot{q}_{2}-r_{1}^{(3)}(t)\right), \quad(70) \\
v_{2}(t) & =r_{2}^{(2)}(t)-\Lambda_{5}\left(f_{3}\left(q_{1}, \dot{q}_{1}\right)+B_{2}\left(q_{1}\right) q_{2}-r_{2}(t)\right) \\
& -\Lambda_{6}\left(f_{4}\left(q_{1}, \dot{q}_{1}, q_{2}\right)+B_{2}\left(q_{1}\right) \dot{q}_{2}-r_{2}^{(1)}(t)\right), \quad(71)
\end{aligned}
$$

and $r_{1}^{(i)}(t)$ and $r_{2}^{(i)}(t)$ denote the $i$-th derivative of $r_{1}(t)$ and $r_{2}(t)$ with respect to time. The tracking errors in the closed loop constrained system satisfy the equations

$$
\begin{aligned}
e_{1}^{(4)}(t)+\Lambda_{4} e_{1}^{(3)}(t)+ & \Lambda_{3} e_{1}^{(2)}(t) \\
& +\Lambda_{2} e_{1}^{(1)}(t)+\Lambda_{1} e_{1}(t)=0, \\
e_{2}^{(2)}(t)+ & \Lambda_{6} e_{2}^{(1)}(t)+\Lambda_{5} e_{2}(t)=0
\end{aligned}
$$


where the position tracking error is defined as

$$
e_{1}(t)=h_{1}\left(q_{1}\right)-r_{1}(t)
$$

and the force tracking error is defined as

$$
e_{2}(t)=\lambda(t)-r_{2}(t)
$$

If $\Lambda_{1}$ through $\Lambda_{6}$ are chosen such that the equations (72) and (73) are Hurwitz, then the tracking error $e_{1}(t)$ for the position outputs and the force tracking error $e_{2}(t)$ approach zero exponentially. In addition, all the internal states of the constrained robot system and the control inputs remain bounded since Assumption 2.2 of Section 2 is satisfied trivially. The control law requires feedback of position and velocity variables of the links of the robot, and the position and velocity variables of the actuator shafts. Force feedback is not necessary. However, if measurement of the contact force $\lambda(t)$ is available it may be included in the feedback loop by replacing the term $f_{3}\left(q_{1}, \dot{q}_{1}\right)+B_{2}\left(q_{1}\right) q_{2}$ in equation (71) by $\lambda(t)$.

\subsection{Constrained robot systems with significant actuator dynamics}

The model of a constrained robot system, described by equations (37)-(40), needs to be modified if the actuator dynamics in the system are significant. In this section, we consider mathematical representations which model a class of constrained robot systems which include the effects of significant actuator dynamics. We consider the case where the joints of the robot are driven by armature controlled direct-current motors. The joints of the robot are assumed to have no flexibility. The model, obtained by modifying equations (37)-(40) to include the significant actuator dynamics, is represented as

$$
\begin{gathered}
M(q) \ddot{q}+H(q, \dot{q})=J^{\mathrm{T}}(q) \lambda+N K_{m} I_{\mathrm{a}}, \\
L \dot{I}_{\mathrm{a}}+R I_{\mathrm{a}}+K_{m} N \dot{q}=u, \\
y=0=\phi(q), \\
z_{1}=h_{1}(q), \\
z_{2}=\lambda,
\end{gathered}
$$

where $q \in R^{n}$ denotes the vector of generalized displacements of the links of the robot, $I_{\mathrm{a}} \in R^{n}$ denotes the vector of currents in the armature circuit of the motors, $L, R$ and $N$ are constant diagonal nonsingular matrices which represent the armature inductances, armature resistances and the gear ratios, respectively. The matrix $K_{m}$ is a nonsingular diagonal matrix which represents the back e.m.f. constants of the motors (which are the same as the torque constants when SI units are used), and $u \in R^{n}$ denotes the vector of control inputs which are the armature voltages of the motors. All the other variables have the same description as in equations (37) $-(40)$.

Suppose $r_{1}(t)$ and $r_{2}(t)$ are vectors of desired position and force trajectories. Equations (74)(78) can be rewritten in a first-order form as

$$
\begin{gathered}
\left(\begin{array}{c}
\dot{q} \\
\ddot{q} \\
I_{\mathrm{a}}
\end{array}\right)=f\left(q, \dot{q}, I_{\mathrm{a}}\right)+g\left(q, \dot{q}, I_{\mathrm{a}}\right) \lambda+h\left(q, \dot{q}, I_{\mathrm{a}}\right) u, \\
y=0=k\left(q, \dot{q}, I_{\mathrm{a}}\right), \\
z=l\left(q, \dot{q}, I_{\mathrm{a}}\right)+m\left(q, \dot{q}, I_{\mathrm{a}}\right) \lambda,
\end{gathered}
$$

where

$$
\begin{gathered}
f\left(q, \dot{q}, I_{\mathrm{a}}\right)=\left[\begin{array}{c}
\dot{q} \\
M^{-1}(q)\left[-H(q, \dot{q})+N K_{m} I_{a}\right] \\
-L^{-1}\left[R I_{\mathrm{a}}+K_{m} N \dot{q}\right]
\end{array}\right], \\
k\left(q, \dot{q}, I_{\mathrm{a}}\right)=\phi(q), \\
g\left(q, \dot{q}, I_{\mathrm{a}}\right)=\left[\begin{array}{c}
0 \\
M^{-1}(q) J^{\mathrm{T}}(q) \\
0
\end{array}\right] . \\
h\left(q, \dot{q}, I_{\mathrm{a}}\right)=\left[\begin{array}{c}
0 \\
0 \\
L^{-1}
\end{array}\right], \\
l\left(q, \dot{q}, I_{\mathrm{a}}\right)=\left[\begin{array}{c}
h_{1}(q) \\
0
\end{array}\right], \quad m\left(q, \dot{q}, I_{\mathrm{a}}\right)=\left[\begin{array}{c}
0 \\
I_{m}
\end{array}\right] .
\end{gathered}
$$

These differential-algebraic equations satisfy assumptions (1)-(3) of Section 1 with a constraint relative degree vector $\left(r_{1}, r_{2}, \ldots, r_{m}\right)$ $=(2,2, \ldots, 2)$ and control relative degree vector $(3,3, \ldots, 3)$. Define

$$
\begin{aligned}
\alpha\left(q, \dot{q}, I_{\mathrm{a}}\right)= & \left(J(q) M^{-1}(q) J^{\mathrm{T}}(q)\right)^{-1} \\
& \times\left[J(q) M^{-1}(q) H(q, \dot{q})\right. \\
& \left.-\dot{J}(q) \dot{q}-J(q) M^{-1}(q) N K_{m} I_{\mathrm{a}}\right] .
\end{aligned}
$$

From equations (9)-(12), $f^{*}, h^{*}, l^{*}$, and $n^{*}$ are given by

$$
\begin{gathered}
f^{*}\left(q, \dot{q}, I_{\mathrm{a}}\right)=f\left(q, \dot{q}, I_{\mathrm{a}}\right)+g\left(q, \dot{q}, I_{\mathrm{a}}\right) \alpha\left(q, \dot{q}, I_{\mathrm{a}}\right), \\
n^{*}\left(q, \dot{q}, I_{\mathrm{a}}\right)=0, \quad h^{*}\left(q, \dot{q}, I_{\mathrm{a}}\right)=h\left(q, \dot{q}, I_{\mathrm{a}}\right), \\
l^{*}\left(q, \dot{q}, I_{\mathrm{a}}\right)=\left[\begin{array}{c}
h_{1}(q) \\
\alpha\left(q, \dot{q}, I_{\mathrm{a}}\right)
\end{array}\right] .
\end{gathered}
$$

Assumptions (4) and (5) of Section 2 are satisfied in this case with $\left(\mu_{1}, \ldots, \mu_{n-m}, \mu_{n-m+1}\right.$, $\left.\ldots, \mu_{n}\right)=(3, \ldots, 3,1, \ldots, 1)$. Now the coordinate change defined by equations (20)-(22) in this case is given by

$$
\begin{aligned}
& \tilde{x}_{1}=\phi(q), \\
& \tilde{x}_{2}=J(q) \dot{q}, \\
& \bar{x}_{1}=h_{1}(q),
\end{aligned}
$$




$$
\begin{gathered}
\bar{x}_{2}=\frac{\partial h_{1}(q)}{\partial q} \dot{q}=P(q) \dot{q}, \\
\bar{x}_{3}=L_{f^{*}}^{2} h_{1}(q)=f_{5}(q, \dot{q})+B_{3}(q) I_{\mathrm{a}}, \\
\bar{x}_{4}=\alpha\left(q, \dot{q}, I_{\mathrm{a}}\right)=f_{6}(q, \dot{q})+B_{4}(q) I_{\mathrm{a}},
\end{gathered}
$$

where $f_{5}, B_{3}, f_{6}, B_{4}$ are defined in the Appendix. Following equations (23)-(27), an equivalent state realization is obtained for the differentialalgebraic equations which is given by

$$
\begin{gathered}
\dot{\bar{x}}_{1}=\bar{x}_{2}, \\
\dot{x}_{2}=\bar{x}_{3}, \\
\dot{x}_{2}=A_{3}\left(q, \dot{q}, I_{\mathrm{a}}\right)+B_{3}(q) L^{-1} u, \\
\dot{x}_{4}=A_{4}\left(q, \dot{q}, I_{\mathrm{a}}\right)+B_{4}(q) L^{-1} u, \\
z_{1}=\bar{x}_{1}, \\
z_{2}=\bar{x}_{4},
\end{gathered}
$$

where

$$
\begin{gathered}
q=\psi\left(\bar{x}_{1}\right), \quad \dot{q}=Q(q) \bar{x}_{2}, \\
I_{\mathrm{a}}=\left[\begin{array}{c}
B_{3}(q) \\
B_{4}(q)
\end{array}\right]^{-1}\left\{-\left(\begin{array}{l}
f_{5}(q, \dot{q}) \\
f_{6}(q, \dot{q})
\end{array}\right)+\left(\begin{array}{l}
\bar{x}_{3} \\
\bar{x}_{4}
\end{array}\right)\right\},
\end{gathered}
$$

and $A_{3}$ and $A_{4}$ are defined in the Appendix. The nonlinear feedback control law defined by equation (28) is now given by

$$
u(t)=L\left[\begin{array}{l}
B_{3}(q) \\
B_{4}(q)
\end{array}\right]^{-1}\left\{-\left(\begin{array}{l}
A_{3}\left(q, \dot{q}, I_{\mathrm{a}}\right) \\
A_{4}\left(q, \dot{q}, I_{\mathrm{a}}\right)
\end{array}\right)+\left(\begin{array}{l}
v_{1}(t) \\
v_{2}(t)
\end{array}\right)\right\}
$$

where

$$
\begin{aligned}
v_{1}(t)=r_{1}^{(3)}(t)- & \Lambda_{1}\left(h_{1}(q)-r_{1}(t)\right) \\
& -\Lambda_{2}\left(P(q) \dot{q}-r_{1}^{(1)}(t)\right) \\
& -\Lambda_{3}\left(f_{5}(q, \dot{q})+B_{3}(q) I_{\mathrm{a}}-r_{1}^{(2)}(t)\right) \\
v_{2}(t)= & r_{2}^{(1)}(t)-\Lambda_{4}\left(f_{6}(q, \dot{q})\right. \\
& \left.+B_{4}(q) I_{\mathrm{a}}-r_{2}(t)\right)
\end{aligned}
$$

and $r_{1}^{(i)}(t)$ and $r_{2}^{(i)}(t)$ denote the $i$-th derivative of $r_{1}(t)$ and $r_{2}(t)$ with respect to time. The tracking errors in the closed loop constrained system satisfy the equations

$$
\begin{gathered}
e_{1}^{(3)}(t)+\Lambda_{3} e_{1}^{(2)}(t)+\Lambda_{2} e_{1}^{(1)}(t)+\Lambda_{1} e_{1}(t)=0, \\
e_{2}^{(1)}(t)+\Lambda_{4} e_{2}(t)=0,
\end{gathered}
$$

where the positive tracking error is defined as

$$
e_{1}(t)=h_{1}(q)-r_{1}(t),
$$

and the force tracking error is defined as

$$
e_{2}(t)=\lambda(t)-r_{2}(t)
$$

If $\Lambda_{1}$ through $\Lambda_{4}$ are chosen such that the equations (82) and (83) are Hurwitz, then the tracking error $e_{1}(t)$ for the position outputs and the force tracking error $e_{2}(t)$ approach zero exponentially. In addition, all the internal states of the constrained robot system and the control inputs remain bounded since Assumption 2.2 of Section 2 is satisfied trivially. The control law requires feedback of position and velocity variables of the links of the robot, and the armature currents of the motors. Force feedback is not necessary. However, if measurement of the contact force $\lambda(t)$ is available it may be included in the feedback loop by replacing the term $f_{6}(q, \dot{q})+B_{4}(q) I_{\mathrm{a}}$ in equation (81) by $\lambda(t)$.

The development in this subsection is also applicable to constrained robot systems driven by a wide class of electrohydraulic servos described in McClamroch (1985) which is given by

$$
\begin{gathered}
M(q) \ddot{q}+H(q, \dot{q})=J^{\mathrm{T}}(q) \lambda+G(q) f_{\mathrm{a}}, \\
k_{h}^{-1} \dot{f}_{\mathrm{a}}+G_{p} f_{\mathrm{a}}+G^{\mathrm{T}}(q) \dot{q}=u, \\
y=0=\phi(q), \\
z_{1}=h_{1}(q), \\
z_{2}=\lambda,
\end{gathered}
$$

where $u$ represents the normalized currents to the servo torque motors, $f_{\mathrm{a}}$ represents the vector of actuator forces. This model includes the effects of fluid compressibility through the constant actuator stiffness $K_{h}$ and the effects of fluid leakage around the actuator pistons through the constant leakage sensitivity matrix $G_{p}$. The matrix $G(q)$ depends on the geometry between the links and the actuators. One can also extend the derivation of the control laws for simultaneous constraint force and position tracking presented here to constrained robot systems which include both the effects of significant actuator dynamics, and the effects of joint flexibility.

\section{CONCLUSION}

In this paper, we have considered the problem of designing a feedback control law so that certain desired outputs track given reference inputs in control systems described by a class of nonlinear differential-algebraic equations. A nonlinear feedback control law has been designed which ensures, under appropriate assumptions, that the tracking error in the closed loop differential-algebraic system approaches zero exponentially. In particular, applications to simultaneous force and position tracking in constrained robot systems have been discussed. Explicit feedback control laws have been derived for constrained robot systems with rigid joints, constrained robot systems with joint flexibility, and constrained robot systems with significant actuator dynamics. 
Acknowledgements - This work was paritally supported by NSF Grant No. MSS-9114630 and by NASA Grant No. NAG-1-1419.

\section{REFERENCES}

Ahmad, S. (1991). Constrained motion (force/position) control of flexible joint robots. Proc. IEEE Conf. Decision and Control, pp. 1397-1402. Brighton, U.K.

Brenan, K. E., S. L. Campbell and L. R. Petzold (1989). Numerical Solution of Initial-value problems in Differential-Algebraic Equations. Elsevier, New York.

Byrnes, C. I. and A. Isidori (1991). Asymptotic stabilization of minimium phase non-linear systems. IEEE Trans. Aut. Control, 36, 1122-1137.

Cai, L. and A. A. Goldenberg (1989). An approach to force and position control of robot manipulators. Proc. IEEE Int. Conf. Robotics and Automation, pp. 86-91. Scottsdale, AZ.

Chen, X. and M. A. Shayman (1992). Dynamics and control of constrained nonlinear systems with application to robotics. Proc. Am. Control Conf., pp. 2962-2966. Chicago, IL.

Cole, A. A. (1989). Control of robot manipulators with constrained motion. Proc. IEEE Conf. Decison and Control, pp. 1657-1658. Tampa, FL.

Isidori, A. (1989). Nonlinear Control Systems, 2nd edn. Springer, Berlin.

Janokowski, K. P. and H. A. Elmaraghy (1992). Dynamic decoupling for hybrid control of rigid/flexible joint robots interacting with the environment. IEEE Trans. Robotics and Automation, 8, 519-534.

Kaprielian, S., J. Turi and L. R. Hunt (1991). Vector input-output linearization for a class of descriptor systems. Proc. IEEE Conf. Decision and Control, pp. 1951-1954. Brighton, U.K.

Krishnan, H. (1992). Control of nonlinear systems with applications to constrained robots and spacecraft attitude stabilization. Ph.D. thesis, Department of Aerospace Engineering, University of Michigan.

Krishnan, H. and N. H. McClamroch (1990). A new approach to position and contact force regulation in constrained robot systems. Proc. IEEE Int. Conf. Robotics and Automation, pp. 1344-1349, Cincinatti, OH.

Krishnan, H. and N. H. McClamroch (1991). Tracking reference inputs in control systems described by a class of nonlinear differential-algebraic equations. Proc. IEEE Conf. Decision and Control, pp. 1796-1801. Brighton, U.K.

Krishnan, H. and N. H. McClamroch (1992). Computation of state realizations for control systems described by a class of linear differential-algebraic equations. Int. J. Control, 55, 1425-1441.

Liu, J. S. (1991). Hybrid position/force tracking for a class of constrained mechanical systems. Systems and Control Lett., 17, 395-399.

McClamroch, N. H. (1985). Displacement control of flexible structures using electrohydraulic servo actuators. ASME J. Dyn. Systems, Measurements and Control, 107, 34-39.

McClamroch, N. H. (1986). Singular systems of differential equations as dynamical models for constrained robot systems. Proc. IEEE Int. Conf. Robotics and Automation, pp. 21-28. San Francisco, CA.

McClamroch, N. H. (1990). Feedback stabilization of control systems described by a class of nonlinear differentialalgebraic equations. Systems and Control Lett., 15, 53-60.

McClamroch, N. H. and D. Wang (1988). Feedback stabilization and tracking of constrained robots. IEEE Trans. Aut Control, 33, 419-426.

Mills, J. K. (1992). Stability and control of elastic-joint robotic manipulators during constrained motion tasks. IEEE Trans Robotics and Automation, 8, 119-125.

Mills, J. K. and A. A. Goldenberg (1989). Force and position control of manipulators during constrained motion tasks. IEEE J. Robotics and Automation, 5, pp. 30-46.

Ramadorai, A. K., T. J. Tarn and A. K. Bejczy (1992). Task-definition, decoupling and redundancy resolution by nonlinear feedback in multirobot object handling. Proc IEEE Int. Conf. Robotics and Automation, pp. 467-474. Nice, France

Reich, S. (1990), On a geometrical interpretation of differential-algebraic equations. Circuits, Systems and Signal Processing, 9, 369-382.

Rheinboldt, W. C. (1984). Differential-algebraic systems as differential equations on manifolds. Math. Computation, 43, 473--482.

Sastry, S. S. and A. Isidori (1989). Adaptive control of linearizable systems. IEEE Trans. Aut. Control, 34, 1123-1131.

Spong, M. W. (1987). Modeling and control of elastic joint robots. ASME J. Dynamic Systems, Measurement and Control, 109, 310-319.

Venkatasubramanian, V., H. Schattler and J. Zaborsky (1990). Global voltage dynamics: study of a generator with voltage control, transmission and matched MW load. Proc. IEEE Conf. Decision and Control, pp. 3045-3056. Honolulu, $\mathrm{HI}$

Wen, J. T. and K. K. Delgado (1992). Motion and force control of multiple robotic manipulators. Automatica, 28 $729-743$.

Yoshikawa, T. (1987). Dynamic hybrid position/force control of robot manipulators-description of hand constraints and calculation of joint deriving force. IEEE J. Robotics and Automation, 3, 386-392.

Yun, X. (1988). Dynamic state feedback control of constrained robot manipulators. Proc. IEEE Conf Decision and Control, pp. 622-626. Austin, TX

\section{APPENDIX}

We first define the variables $f_{1}, B_{1}, f_{2}, B_{2}, f_{3}$ and $f_{4}$ used in Section 3.2. Suppressing the arguments, we denote $M\left(q_{1}\right)$ as $M, J\left(q_{1}\right)$ as $J, P\left(q_{1}\right)$ as $P$, and $H\left(q_{1}, \dot{q}_{1}\right)$ as $H$. The variables are defined as

$$
\begin{aligned}
& f_{3}\left(q_{1}, \dot{q}_{1}\right)=\left(J M^{-1} J^{\mathrm{T}}\right)^{-1}\left(J M^{-1} H-\dot{J}_{\dot{q}_{1}}+J M^{-1} K_{2} q_{1}\right), \\
& B_{2}\left(q_{1}\right)=-\left(J M^{-1} J^{\mathrm{T}}\right)^{-1} J M^{-1} K_{2}, \\
& f_{1}\left(q_{1}, \dot{q}_{1}\right)=\dot{P}_{\dot{q}_{1}-} P M^{-1}\left(H+K_{2} q_{1}\right)+P M^{-1} J^{\mathrm{T}} f_{3}, \\
& B_{1}\left(q_{1}\right)=P M^{-1}\left(K_{2}+J^{\mathrm{T}} B_{2}\right), \\
& f_{2}\left(q_{1}, \dot{q}_{1}, q_{2}\right)= \\
& \frac{\partial\left(f_{1}+B_{1} q_{2}\right)}{\partial q_{1}} \dot{q}_{1}+\frac{\partial f_{1}}{\partial \dot{q}_{1}} \\
& \times\left[M^{-1}\left(-H+K_{2}\left(q_{2}-q_{1}\right)\right)\right. \\
&\left.+M^{-1} J^{\mathrm{T}}\left(f_{3}+B_{2} q_{2}\right)\right], \\
& f_{4}\left(q_{1}, \dot{q}_{1}, q_{2}\right)= \frac{\partial\left(f_{3}+B_{2} q_{2}\right)}{\partial q_{1}} \dot{q}_{1}+\frac{\partial f_{3}}{\partial \dot{q}_{1}} \\
& \times\left[M^{1}\left(-H+K_{2}\left(q_{2}-q_{1}\right)\right)\right. \\
&\left.+M^{-1} J^{\mathrm{T}}\left(f_{3}+B_{2} q_{2}\right)\right] .
\end{aligned}
$$

The variables $A_{1}$ and $A_{2}$ in Section 3.2 are defined as

$$
\begin{aligned}
A_{1}\left(q_{1}, \dot{q}_{2}, \dot{q}_{2}\right) & \\
= & \frac{\partial\left(f_{2}+B_{1} \dot{q}_{2}\right)}{\partial q_{1}} \dot{q}_{1}+\frac{\partial f_{2}}{\partial q_{2}} \dot{q}_{2}-B_{1} M_{2}^{-1} K_{2}\left(q_{2}-q_{1}\right) \\
& +\frac{\partial f_{2}}{\partial \dot{q}_{1}}\left[M^{-1}\left(-H+K_{2}\left(q_{2}-q_{1}\right)\right)+M^{-1} J^{\mathrm{T}}\left(f_{3}+B_{2} q_{2}\right)\right], \\
A_{2}\left(q_{1}, \dot{q}_{1}, q_{2}, \dot{q}_{2}\right) & \\
= & \frac{\partial\left(f_{4}+B_{2} \dot{q}_{2}\right)}{\partial q_{1}} \dot{q}_{1}+\frac{\partial f_{4}}{\partial q_{2}} \dot{q}_{2}-B_{2} M_{2}^{-1} K_{2}\left(q_{2}-q_{1}\right) \\
& +\frac{\partial f_{4}}{\partial \dot{q}_{1}}\left[M^{-1}\left(-H+K_{2}\left(q_{2}-q_{1}\right)\right)+M^{-1} J^{\mathrm{T}}\left(f_{3}+B_{2} q_{2}\right)\right]
\end{aligned}
$$

Next, the variables $f_{5}, B_{3}, f_{6}, B_{4}$ in Section 3.3 are defined. Suppressing the arguments, we denote $M(q)$ as $M, J(q)$ as 
$J, P(q)$ as $P$, and $h(q, \dot{q})$ as $H$. The variables are defined as

$$
\begin{gathered}
f_{6}(q, \dot{q})=\left(J M^{-1} J^{\mathrm{T}}\right)^{-1}\left(J M^{-1} H-\dot{j} \dot{q}\right), \\
B_{4}(q)=-\left(J M^{-1} J^{\mathrm{T}}\right)^{-1} J M^{-1} N K_{m}, \\
f_{5}(q, \dot{q})=\dot{P} \dot{q}-P M^{-1}\left(H-J^{\mathrm{T}} f_{6}\right), \\
B_{3}(q)=P M^{-1} J^{\mathrm{T}} B_{4}+P N K_{m} .
\end{gathered}
$$

The variables $A_{3}$ and $A_{4}$ in Section 3.3 are defined as $A_{3}\left(q, \dot{q}, I_{\mathrm{a}}\right)$

$$
=\frac{\partial\left(f_{5}+B_{3} I_{\mathrm{a}}\right)}{\partial q} \dot{q}-B_{3} L^{-1}\left(R I_{\mathrm{a}}+K_{m} N \dot{q}\right)
$$

$$
+\frac{\partial f_{5}}{\partial \dot{q}}\left[M^{-1}\left(-H+N K_{m} I_{\mathrm{a}}\right)+M^{-1} J^{\mathrm{T}}\left(f_{6}+B_{4} I_{a}\right)\right]
$$

$A_{4}\left(q, \dot{q}, I_{\mathrm{a}}\right)$

$$
=\frac{\partial\left(f_{6}+B_{4} I_{\mathrm{a}}\right)}{\partial q} \dot{q}-B_{4} L^{-1}\left(R I_{\mathrm{a}}+K_{m} N \dot{q}\right)
$$

$+\frac{\partial f_{6}}{\partial \dot{q}}\left[M^{-1}\left(-H+N K_{m} I_{\mathrm{a}}\right)+M^{-1} J^{\mathrm{r}}\left(f_{6}+B_{4} I_{\mathrm{a}}\right)\right]$. 